\title{
Study and Simulation of Electromagnetic Energy Recovery for Semi - active Suspension
}

\author{
Zhijun Guo ${ }^{1, a}$,Yichao Chen ${ }^{1, b}$ \\ ${ }^{1}$ Vehicle \& Transportation Engineering Institute \\ Henan University of Science and Technology \\ Luoyang, China \\ aemail : gzhj1970@163.com, bemail : chenyichao002@126.com
}

\begin{abstract}
Keywords: Semi-active suspension; Electromagnetic type; Energy recovery; simulation
Abstract. Aiming at the problem that the existing semi-active suspension system needs external power supply, the electromagnetic energy recovery device for semi-active suspension is proposed. Based on the analysis of the structure and working principle of the energy recovery device, the energy recovery model and the starting resistance model are constructed. Under the premise that satisfying the electricity demand of the type of semi-active suspension and the usual working conditions (i.e. B-level road and economic speed $(36 \mathrm{~km} / \mathrm{h})$ ), the influence that the gear radius on the output energy and the starting resistance of the designed energy recovery device is studied, and the basic structural parameters of the model are determined. The output energy of the energy recovery device was simulated by MATLAB software, and the influence of the vehicle speed (36 $\mathrm{km} / \mathrm{h} \sim 60 \mathrm{~km} / \mathrm{h}$ ) on the output power was analyzed. The result shows that the output current of the energy recovery device is about $1.53 \mathrm{~A}$ under the condition of economic speed, indicating that the energy recovery device can meet the demand of a certain semi-active suspension (1.4A) under the premise and has excess power for charging.
\end{abstract}

\section{Introduction}

The damping coefficient or stiffness of the suspension constituent elements is controlled by a semi-active suspension within a certain range. Compared with active suspension, semi-active suspension has lower energy consumption, simple structure, low cost, high reliability, and its performance is similar to that of active suspension [1]. Generally, the static load of automobile body is assumed by the elastic component of suspension, so it is much more difficult to implement stiffness control in semi-active suspension than damping control. At present, the research of semi-active suspension is mostly confined to the research of damping coefficient control [2]. MR damper is a kind of intelligent damper, which has the advantages of fast response, continuous damping, low energy consumption and so on. However, in MR damper operation, external power supply is needed to provide power to achieve continuous damping. If external power is interrupted, its reliability is greatly affected. Therefore, how to transform the mechanical energy generated by the vibration of the frame into the electrical energy through the energy recovery device which provides power for MR dampers to eliminate their dependence on external power supplies has become one of the main research directions [3-8].

Scholars have studied semi-active suspension based on electromagnetic energy feeding. Chen, et al [4] and Ski, B et al [5] used the linear permanent magnetic induction energy collection system to study the self powered of MR damper, carried on the experiment and got a good result. However, the direction of the permanent magnet field of the energy recovery device is in a certain angle with its axis of the generator coil, accordingly, this reduces the utilization ratio of magnetic flux. Jiang Xuezheng et al [6] and Yue Xu et al [7] studied the structural design and electrical energy collection of the self powered MR damper respectively, has achieved good results. However, there is no proper solution for how to increase magnetic flux. In the paper [8], the rotating permanent magnetic induction energy recovery device was used to improve the self generating power of the MR damper, the experiments were carried out and the expected effect was obtained. However, the effect of the 
designed energy recovery device on the damping force of MR damper is not considered when it is started. Accordingly, it is important to study how to improve the utilization of magnetic flux and study the starting resistance.

For these reasons, an electromagnetic energy recovery device (hereinafter referred to as energy recovery device) is proposed. The energy recovery device adopts a rotating structure, and the permanent magnet induction magnetic field vertically cuts into the power generating coil, and the induction magnetic field direction is parallel to the axis of the power generation coil. Based on the analysis of the structure and working principle of the designed energy recovery device, the generation model and the starting resistance model are constructed. The performance of the designed energy recovery device is simulated by using MATLAB/Simulink software, and the influence of its power generation characteristics and starting resistance is analyzed.

\section{Structure and working principle of energy recovery device}

The Fig. 1 is a sketch map of suspension motion. Wherein, the rotating shaft is arranged on the frame with the energy recovery device. The rack is fixed to the axle, the gear is fixed on the rotating shaft. The gear and the rack form the transmission component. The vertical relative movement of the frame and the axle is converted into a rotational relative movement by the transmission component when the frame vibrates. The Fig. 2 is a schematic diagram of an energy recovery device.

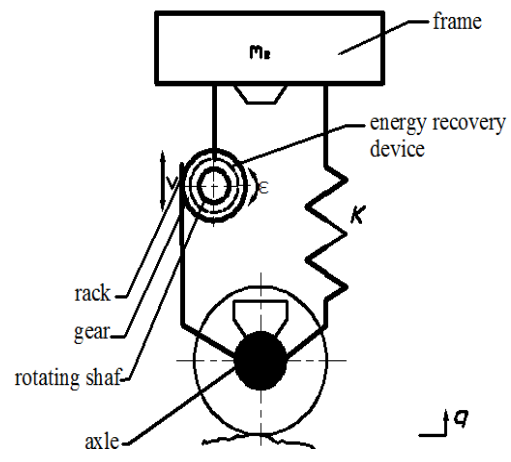

Fig. 1 Sketch map of suspension motion

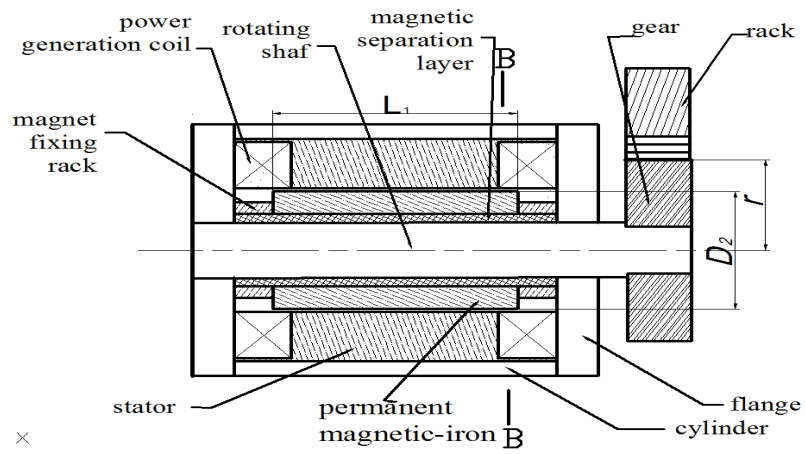

Fig. 2 Schematic diagram of an energy recovery device

The Fig. 3 is a sectional view of The Fig. 2. Wherein, the magnet fixing rack is fixedly connected with the rotating shaft. The permanent magnetic-irons are evenly arranged radialy along the rotating shaft. A single-phase winding composed of a plurality of power generation coils in reverse series is fixed in the stator groove. The material of permanent magnetic-irons is a $\mathrm{Nd}-\mathrm{Fe}-\mathrm{B}$ magnet (model N50), the magnetic field direction is distributed radially, and the polarity of the induction surface of the adjacent two permanent magnetic-irons is opposite. The material of power generation coils is conventional enameled wire. The magnet fixing frame is

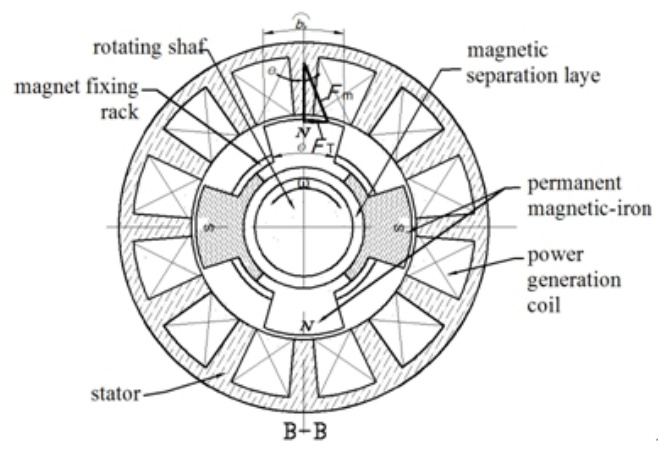

Fig. 3 Sectional view of The Fig. 2 made of silicon steel. Non-magnetic material is used in the magnetic separation layer. When the frame vibrates, the power generation coil in the stator groove rotates relative to the permanent magnetic-irons on the rotating shaft. At the same time, the permanent magnetic-irons induced magnetic field vertically cuts into the power generation coil, so that the magnetic flux inside the generator coil will be change. According to Faraday's law of electromagnetic induction, both ends of the wire of the closed coil will generate an induced electromotive force that changes when the flux passes through the closed coil. The generated electric energy is rectified and stored in a storage battery to supply electric power to the excitation coil. 


\section{Energy recovery model and parameter determination}

\section{Energy recovery model}

When the rotating shaft drives the permanent magnetic-irons to rotate relative to the power generation coils at an angular speed of $\omega$, the power generation coils generates electromotive force because of the change of the magnetic flux passing through the inner coil. The process agrees with Faraday's law of electromagnetic induction. We define the induced electromotive force of the generator coils as $e$. The electromotive force $e$ is

$$
e=-N \frac{\mathrm{d} \phi}{\mathrm{d} t}=-\frac{\mathrm{d} \psi}{\mathrm{d} t}
$$

where $e$ is the electromotive force of the generator coils, $\phi$ is the flux of a single turn coil of a generator coil, $N$ is the Number of turns of a coils, $\psi$ is the total magnetic flux in a power coils, i.e. $\psi=N \cdot \phi$.

When the frame vibrates, the rotating shaft drives the permanent magnetic-irons to do positive and negative rotation motion, the variation of the magnetic flux in the coil of the generator conforms to the sine law, i.e. $\phi$ is expressed as

$$
\phi=\phi_{m} \sin \omega t
$$

where $\phi_{m}$ is the maximum magnetic flux through a generator coil. Introduce (2) into (1), the electromotive force $e$ can be rewritten as

$$
e(t)=-N \frac{\mathrm{d} \phi}{\mathrm{d} t}=-N \omega \phi_{m} \sin \left(\omega t-\frac{\pi}{2}\right)
$$

The rack speed is defined as $v$, the radius of the gear is defined as $r$. The motion relation of the rack and pinion drive component is expressed as $v=r \cdot \omega$. Introduce $v=r \cdot \omega$ into (3), the relation between the electromotive force $e$ of the generator coil and the rack movement speed $v$ can be expressed as

$$
e(t)=-N \frac{v}{r} \phi_{m} \sin \left(\frac{v}{r} t-\frac{\pi}{2}\right)
$$

According to the principle of equal current thermal effect in the same time, the effective value of the induced electromotive force generated by the generator coils $E$ can be written as

$$
E=\sqrt{\frac{1}{T} \int_{0}^{T} e^{2}(t) d t}
$$

If the energy recovery device is connected to the load, the power output outward can be expressed as

$$
P=\left(\frac{E}{R+R_{1}}\right)^{2} R_{1}
$$

Where $P$ is output power, $R_{1}$ is the load resistance, $R$ is the generator coils resistance.

Analyzed (4), (5) and (6),we can see that the effective electromotive force $E$ and the effective power $P$ of the external output of the energy recovery device are directly proportional to the motion velocity $v$ of the rack, and increase with the increase of $v$. The $E$ and the $P$ are inversely related to the radius of the gear, and decrease with the increase of $r$.

\section{Starting resistance model}

When the frame vibrates, the permanent magnetic-irons rotate in a positive and negative rotation with the rotating shaft, and the stroke of the permanent magnetic-irons conform to the sine law. 
Relative to the power generation coil, the permanent magnetic-irons are in a discontinuous start stop state.

When the energy recovery device is not started, the permanent magnetic-irons' magnetic pole fixed on the rotating shaft attracts the stator teeth through the air gap. The attraction of the permanent magnetic-irons' magnetic pole to the stator teeth is represented by $F_{m}$, the $F_{m}$ can be expressed as [9]

$$
F_{m}=\frac{B_{\delta}^{2}}{2 \mu_{0}} A_{m}
$$

where $B_{\delta}$ is gap flux density, $\mu_{0}$ is absolute permeability of vacuum, $\mu_{0}=4 \pi \cdot 10^{-7} \mathrm{H} / \mathrm{m}, A_{m}$ is the area of permanent magnetic-irons' magnetic pole .The $A_{m}$ can be written as

$$
A_{m}=b_{a} L_{1}
$$

where $b_{a}$ is the magnetic arc length of permanent magnetic-irons, $L_{1}$ is the effective length of magnetic pole.

When the energy recovery device is not started, the permanent magnet pole is aligned with the stator teeth to be answered. When the energy recovery device starts, the rotating shaft rotates. The angle between permanent magnet radial and the direction of the attraction $F_{m}$ is $\theta$, as shown in Figure 3. The farther the magnet pole moves away from its aligned stator teeth, the greater the angle of $\theta$, The $F_{T}$ represents the tangential force of the attraction $F_{m}$ on the circumference, so the $F_{T}$ is the rotor resistance. The angle between the direction of force and the direction of displacement is analyzed, when $\theta=90^{\circ}, F_{m}=F_{T}$. The rotation angle of the rotor can be represented by the arc angle corresponding to the length of the permanent magnet arc, i.e. the $\theta$ can be expressed as

$$
\theta=\frac{360^{\circ}}{\pi D_{2}} b_{a}
$$

where $D_{2}$ is the rotor outer diameter.

Introduce (8) and (9) into (7), the rotor resistance $F_{T}$ can be written as

$$
F_{T}=F_{m} \sin \theta=\frac{B_{\delta}^{2} b_{a} L_{1}}{2 \mu_{0}} \sin \frac{360^{\circ}}{\pi D_{2}} b_{a}
$$

When $m \cdot e$ permanent magnetic-irons' poles is fully aligned with its corresponding aligned stator, the $F_{T}$ can be rewritten as

$$
F_{T}=m e T_{m} \sin \theta=m e \frac{B_{\delta}^{2} b_{a} L_{1}}{2 \mu_{0}} \sin \frac{360^{\circ}}{\pi D_{2}} b_{a}
$$

where $m$ is the phase number of permanent magnet energy recovery device, $e$ is the number of slots per phase per pole.

We define $l$ as the distance between poles of the energy recovery device, $p$ as the pole count of the energy recovery device. On the basis of $\pi \bullet D_{2}=2 p \bullet l$ and $b_{a}=(0.55 \sim 0.75) l$, (9) can be written as [9]

$$
\theta=\frac{360^{\circ}}{2 p l}(0.55-0.75) l
$$

We define the starting torque of the energy recovery device as $M_{T}$. Introduce (12) into (11), and according to $M_{T}=D_{2} \bullet F_{T} / 2$, the $M_{T}$ can be rewritten as

$$
M_{T}=\frac{D_{2}}{2} F_{T}=m e \frac{D_{2} B_{\delta}^{2} b_{a} L_{1}}{2 \mu_{0}} \sin \left[\frac{360^{\circ}}{2 p}(0.55 \sim 0.75)\right]
$$

In order to keep the start margin, expand the value of (0.55 0.75)as 1 . The $M_{T}$ can be expressed as 


$$
M_{T}=m e \frac{D_{2} B_{\delta}^{2} b_{a} L_{1}}{2 \mu_{0}} \sin \left(\frac{360^{\circ}}{2 p}\right)
$$

We define $F_{z}$ as the starting resistance of the energy recovery device, the $F_{z}$ can be written as

$$
F_{Z}=\frac{M_{T}}{r}=m e \frac{D_{2} B_{\delta}^{2} b_{a} L_{1}}{2 r \mu_{0}} \sin \left(\frac{360^{\circ}}{2 p}\right)
$$

According to (15), we can see that the starting resistance $F_{z}$ is inversely proportional to the gear radius $r$, and the bigger the $r$, the smaller the $F_{z}$.

\section{Analysis of gear radius}

According to (4) and (15), we can see that the energy and the starting resistance produced by the energy recovery device are inversely proportional to the radius of the gear. The electric energy and the starting resistance increase with the decrease of the gear radius.

However, the starting resistance has a certain influence on the damping force demand of semi-active suspension, and then affects the performance of semi-active suspension. The more bigger the starting resistance, the more stronger the starting resistance's effect on semi-active suspension performance. Therefore, it is necessary to optimize the gear radius to reduce the influence of the gear radius on the performance of semi-active suspension when meeting the electricity demand of the semi-active suspension.

The influence of gear radius on the energy output of the energy recovery model is investigated under the usual working conditions of a MR damper (i.e., B-class road and $u=10 \mathrm{~m} / \mathrm{s}$ economic speed). Under the condition of satisfying the power demand of the damper (excitation current $0 \mathrm{~A} \sim 1.4 \mathrm{~A}$ and excitation coil resistance $R_{1}=1.26 \Omega$ ), the percentage of starting resistance and maximum damping force $(1100 \mathrm{~N})$ is observed. And the suitable gear radius is optimized and determined. In order to meet the basic demand of the damper, the basic structural parameters of the energy recovery device are determined. The basic structure parameters are shown in Table 1.

Table 1. Basic structure parameters the energy recovery device

\begin{tabular}{lccc}
\hline \multicolumn{1}{c}{ parameter } & value & parameter & value \\
\hline magnetic arc length/ $b_{a}$ & $0.015[\mathrm{~mm}]$ & number of slots per phase per pole/ $e$ & 2 \\
gap flux density/ $B_{\delta}$ & $0.5[\mathrm{~T}]$ & excitation coil resistance/ $R_{1}$ & $1.26[\Omega]$ \\
absolute permeability of vacuum $/ \mu_{0}$ & $4 \pi \cdot 10^{-7}[\mathrm{H} / \mathrm{m}]$ & pole count $/ p$ & 2 \\
effective length of magnetic pole/ $L_{1}$ & $80[\mathrm{~mm}]$ & Number of turns of a coils $/ N$ & 1630 \\
rotor outer diameter/ $D_{2}$ & $48[\mathrm{~mm}]$ & maximum magnetic flux $/ \Phi_{m}$ & $0.0012[\mathrm{~Wb}]$ \\
phase number/ $m$ & 2 & &
\end{tabular}

The relationship between the effective current and the gear radius is obtained by simulation, as shown in Figure 4. The relation between the starting resistance and the radius of the gear is obtained by simulation, as shown in figure 5 .

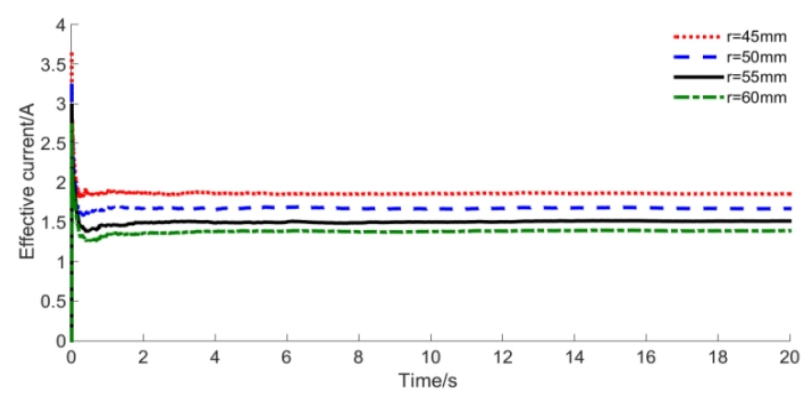

Fig. 4 The relation curve of effective current and gear radius

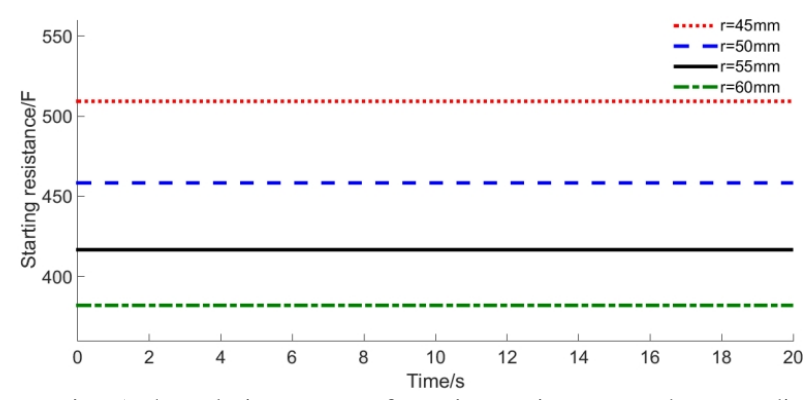

Fig. 5 The relation curve of starting resistance and gear radius 
As shown in Figure 4, the effective current gear radius relation curve is known. Under the working condition that In the B-class white noise pavement and at the speed of $u=10 \mathrm{~m} / \mathrm{s}$, when the gear radius is $45 \mathrm{~mm}$, the effective current flowing through the load is about 1.87A, when the radius of the gear is $50 \mathrm{~mm}$, the effective current flowing through the load is about $1.68 \mathrm{~A}$, when the radius of the gear is $55 \mathrm{~mm}$, the effective current flowing through the load is about $1.53 \mathrm{~A}$, when the radius of the gear is $60 \mathrm{~mm}$, the effective current flowing through the load at about 1.38A. The analysis shows that the effective current flowing through the load decreases with the increase of the gear radius.

As shown in Figure 5. When the gear radius is $45 \mathrm{~mm}$, the starting resistance $F_{\mathrm{z}}$ is about $508 \mathrm{~N}$, which is $46 \%$ of the maximum damping force. When the gear radius is $50 \mathrm{~mm}$, the starting resistance $F_{\mathrm{z}}$ is about $458 \mathrm{~N}$, which is $41 \%$ of the maximum damping force. When the gear radius is $55 \mathrm{~mm}$, the starting resistance $F_{\mathrm{z}}$ is about $416 \mathrm{~N}$, which is $37 \%$ of the maximum damping force. When the gear radius is $60 \mathrm{~mm}$, the starting resistance $F_{\mathrm{z}}$ is about $381 \mathrm{~N}$, which is $34 \%$ of the maximum damping force. It can be seen that with the increase of the gear radius, the starting resistance decreases gradually, and the percentage of the maximum damping force decreases. The starting resistance's effect on semi-active suspension performance is reduced.

Based on the analysis of the data that showing the starting resistance-gear radius' relationship and the effective current-gear radius' relationship, the gear radius $r=55 \mathrm{~mm}$ is determined under the normal working condition of magneto rheological damper.

\section{Simulation analysis}

According to the energy recovery model built in the second part, a simulation model is built in the MATLAB/Smulink environment, as shown in figure 6. The white noise road signal is input into the excitation module, and the relation between the vehicle speed and the output energy of the energy recovery device is analyzed.

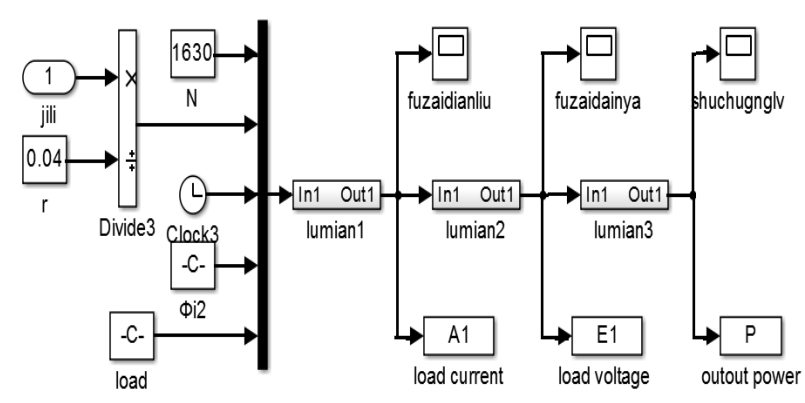

Fig. 6 Energy simulation model

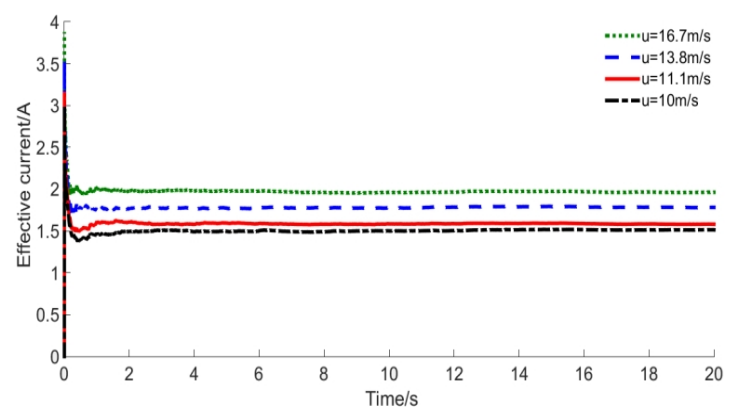

Fig. 7 the relation curve of Effective current and speed

In simulation, the white noise road signal is used as input to simulate the B-class road excitation. We define the road roughness coefficient as $G_{q}\left(n_{0}\right)$, The value of the $G_{q}\left(n_{0}\right)$ in the pavement module is $64 \times 10^{-6} \mathrm{~m}^{3}$. The noise intensity of white noise module is 0.5 . The load module indicates the excitation coil resistance $R_{1}=1.26 \Omega$. On the premise that the gear radius is $r=55 \mathrm{~mm}$, the speed values $\mathrm{u}=10 \mathrm{~m} / \mathrm{s}, 11.1 \mathrm{~m} / \mathrm{s}, 13.8 \mathrm{~m} / \mathrm{s}$ and $16.6 \mathrm{~m} / \mathrm{s}$ are input in the speed module respectively, and the current characteristic curve of load $\mathrm{R}=1.26 \Omega$ is obtained, as shown in Fig. 7.

From the simulation curve of figure 7 , it can be seen that under the condition of white noise, the effective current of the flow through the load is about $1.53 \mathrm{~A}$ when the speed $\mathrm{u}$ is $10 \mathrm{~m} / \mathrm{s}$, the effective current of the flow through the load is about $1.59 \mathrm{~A}$ when the speed $\mathrm{u}$ is $11.1 \mathrm{~m} / \mathrm{s}$, the effective current of the flow through the load is about $1.79 \mathrm{~A}$ when the speed $\mathrm{u}$ is $13.8 \mathrm{~m} / \mathrm{s}$, the effective current of the flow through the load is about 1.97A when the speed $u$ is $16.6 \mathrm{~m} / \mathrm{s}$. The analysis shows that the effective current through the load increases with the increase of the speed.

Through the analysis of the data which express effective current-speed's relationship, we found that the designed energy recovery device model can meet the electricity demand of a magneto rheological damper under normal operating conditions. 


\section{Conclusion}

This paper proposes an electromagnetic energy recovery device for semi-active suspension. The electromagnetic energy recovery adopts a permanent magnetic induction field to vertically cut in the generator coil structure, so that the direction of the induced magnetic field is parallel to the axis of the generator coil, and the utilization ratio of the permanent magnetic induction flux is increased. The relationship between the starting resistance with the effective current and the gear radius of the energy recovery device is analyzed and the radius of the gear is determined. The energy characteristic analysis of the energy recovery device shows that under the general working conditions that B-class road and economic speed $(\mathrm{u}=10 \mathrm{~m} / \mathrm{s})$, the power demand of a MR damper can be satisfied and the electricity is rich for charging.

\section{Acknowledgements}

This work was financially supported by the National High Technology Research and Development Program (2012AA111204). We express our thanks.

\section{References}

[1] Liao Changrong. Research on magnetorheological damper of automotive suspension system, D. Chongqing: Chongqing University, 2001.

[2] Yu Miao. Research on vehicle magnetorheological semi-active suspension control system, D .Chongqing: Chongqing University, 2003.

[3] Yu fan, Zhang Yongchao. Active suspension technology of energy fed vehicles, J. Transactions of the Chinese Society for Agricultural Machinery, Vol. 41(2010), p. 1-6.

[4] Chao Chen , Wei-Hsin Liao. A self-sensing magnetorheological damper with power generation, J . Smart Mater. Struct, 21 (2012) 025014 (14pp).

[5] Ski B. Energy-harvesting linear MR damper: prototyping and testing, J . Smart Mater. Struct, 23 (2014) 035021 (15pp).

[6] Jiang Xuezheng, Hu Hongsheng and Wang Jiong. Study of magnetorheological damper based on electromagnetic induction energy harvesting, J . Journal of Vibration and Shock, Vol. 31(2012), p. 11-15.

[7] Yue Xu, Liu Shulian, Yang Likang, Sun Haijie. Design and Simulation of energy fed magnetorheological damper, J Journal of Mechanical \& Electrical Engineering, Vol. 32(2015), p. $1038-1043$.

[8] Ki Sum Chu, Li Zou and Wei-Hsin Liao. A mechanical energy harvested magnetorheological damper with linear-rotary motion converter, Sensors and Smart Structures Technologies for Civil, Mechanical, and Aerospace Systems 2016. J. Las Vegas, NV. Vol. 9803, 980309(2016), p.1-10.

[9] Su Shaoyu, Gao Hongxia.Yongci Fadianjijili Sheji Jiyingyong , Second ed(China Machine Press, Beijing 2015). 